\title{
Article \\ Microphone Array for Speaker Localization and Identification in Shared Autonomous Vehicles
}

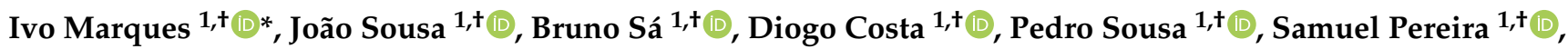 \\ Afonso Santos ${ }^{1,+(\mathbb{D}}$, Carlos Lima ${ }^{1,+}+\mathbb{C}$, Niklas Hammerschmidt ${ }^{2}$, Sandro Pinto ${ }^{1,+(\mathbb{C})}$, and Tiago Gomes ${ }^{1,+(\mathbb{C})}$ \\ 1 Centro ALGORITMI, Universidade do Minho, Portugal; \{ivo.marques, joao.sousa, bruno.sa, diogo.costa, \\ pedro.sousa, afonso.santos, clima, sandro.pinto,mr.gomes\}@dei.uminho.pt \\ 2 Bosch Car Multimedia, Braga, Portugal; niklas.hammerschmidt@pt.bosch.com \\ * Correspondence: ivo.marques@dei.uminho.pt; Tel.: +351-253510180 (I.M.) \\ + Current address: Centro ALGORITMI, Escola de Engenharia - Universidade do Minho, 4800-058 \\ Guimarães, Portugal
}

\begin{abstract}
With the current technological transformation in the automotive industry, autonomous vehicles are getting closer to the Society of Automative Engineers (SAE) automation level 5. This level corresponds to the full vehicle automation, where the driving system autonomously monitors and navigates the environment. With SAE-level 5, the concept of a Shared Autonomous Vehicle (SAV) will soon become a reality and mainstream. The main purpose of an SAV is to allow unrelated passengers to share an autonomous vehicle without a driver/moderator inside the shared space. However, to ensure their safety and well-being until they reach their final destination, it is required an active monitoring of all passengers. In this context, this article presents a microphone-based sensor system that is able to localize sound events inside an SAV. The solution is composed of a Micro-Electro-Mechanical System (MEMS) microphone array with a circular geometry connected to an embedded processing platform that resorts to Field-Programmable Gate Array (FPGA) technology to successfully process in hardware the sound localization algorithms.
\end{abstract}

Keywords: Shared Autonomous Vehicle (SAV); Field-Programmable Gate Array (FPGA); Microphone Array; Sound Source Localization.

\section{Introduction}

The concept of shared mobility, such as car-sharing or rent-by-the-hour vehicles, where passengers can partially or totally share the same trip, tend to become a common practice present in modern societies [1]. At the same time, the technological advances in the automotive industry have been highly contributing for the future of autonomous vehicles in a sustainable urban mobility scenario [2,3]. The merging between the autonomous driving and the shared mobility trends, resulted in the emergence of the concept of Shared Autonomous Vehicle (SAV) [1,4], which enables unrelated passengers to share the same vehicle during their trips. The adoption of SAV will completely change the current paradigm of shared rides and it will surely contribute for a more sustainable and affordable way of passenger mobility in urban areas.

In current ride-share or taxi services, despite the driver moderating the activities inside the common space, some companies such as Uber, Lyft, and Didi, have reported several safety problems between passengers and drivers [5]. There have been records of harassment, assault and robbing passengers, and unfortunately no strict measures could be taken since the company cannot have full control over the passengers, drivers, vehicles or rides. In the context of an SAV, and since the vehicle will not require the driver's control, these problems can become worse since the absence of a moderator can leave the vehicle vulnerable to misuse and inappropriate behaviour between passengers, causing several consequences both for the occupants and the car. 
Being the safety of all occupants a major concern, it is crucial to develop solutions to ensure a normal ride during shared trips. Current trends aim at equipping an SAV with sensor-based monitoring solutions to analyze and identify several situations inside the vehicle's shared space, e.g., driver's and passenger's behaviour, violence between occupants, vandalism, assaults, etc., to trigger safety measures. Current solutions that monitor the activity inside the vehicle are mostly video-based systems [6-8]. However, video-only solutions are not able to capture all the surroundings as they have a limited field of view, making hard the classification and detection of all human actions inside the vehicle. Thus it is almost mandatory to collect audio events inside the shared space [9]. Current audio-only sensor systems use microphone arrays to localize different sound sources $[10,11]$. Other hybrid approaches combine microphone arrays with video cameras combined with facial recognition techniques to localize and detect audio sources, which can be used to monitor the SAV $[12,13]$. However they sometimes require complex sensor fusion systems with high processing capabilities.

With the challenge of creating an audio-only sensor system to localize and identify speakers without requiring a video-based solution, this work presents an embedded, costeffective, low-power, and real-time microphone array solution for speaker localization and identification that can be used inside an SAV. To accelerate the processing tasks, the sensor system resorts to Field-Programmable Gate Array (FPGA) technology to deploy dedicated processing modules in hardware to interface, acquire, and compute data from different microphones [14-19]. Moreover, the processing system provides a Robot Operating System (ROS) interface to make data available to other high-level applications (for the identification and classification of audio events) or to other sensor fusion systems. Finally, and since the system deals with sensitive data, we have deployed the processing systems over a static partitioning hypervisor to guarantee data security and prevent unwanted accesses to private information. This work was developed in partnership with Bosch Car Multimedia Portugal, S.A., and contributes to the state of the art with:

1) a microphone array system to monitor sound events inside an SAV;

2) a hardware-based system with data acquisition and format conversion, i.e., Pulse Density Modulated (PDM) to Pulse Code Modulated (PCM) to interface the microphone array;

3) hardware-accelerated algorithms to localize different sound sources.

\section{Sensor System Architecture}

The sensor system's architecture with all modules and their respective interactions is depicted in Figure 1. It is mainly divided into three main blocks: (1) the microphone array, (2) the processing platform, and (3) the ROS environment.

\section{Microphone Array}

The microphone array includes seven microphones in a Uniform Circular Array (UCA) geometry. The output of each microphone is a PDM signal containing the necessary information to localize and separate the sound sources. The microphone's board is connected to the processing platform through an FPGA Mezzanine Card (FMC) interface, which is used to power the board, get data from the microphones, and provide the clock signal to generate the data output.

\section{Processing platform}

The processing platform is responsible to acquire and process in real-time data retrieved from the microphone array module. It consists of the Xilinx Zynq UltraScale+, which includes a MultiProcessor System on a Chip (MPSoC) with Programmable Logic (PL) FPGA technology. This allows the acceleration in hardware of the microphone array interface and the source localization algorithms. On the PL side, the PDM-to-PCM module is responsible to convert the audio signal from the PDM to PCM format and to apply filtering steps to prevent signal aliasing and spatial-aliasing [20]. The conversion 


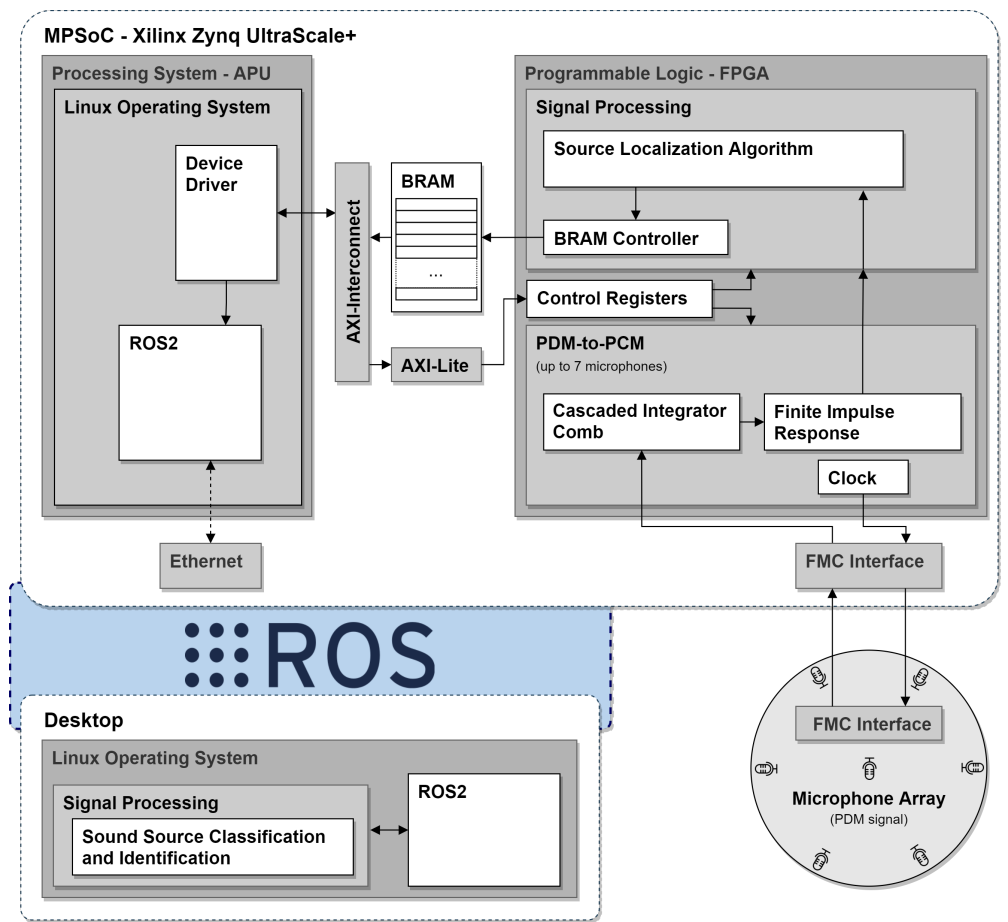

Figure 1. Sensor system architecture.

from PDM to PCM is required by the sound processing algorithm in the next hardware block. This step is performed in the Signal Processing module, where a set of calculations and bit operations in the FPGA are executed to estimate the Direction of Arrival (DoA) of the sound sources. The processed data, which contains the estimated DoA and the acquired signal, is sent to the Processing System (PS) through the Advanced eXtensible Interface (AXI) protocol in the Advanced Microcontroller Bus Architecture (AMBA) bus. On the PS side, the data is collected through a standard device driver supported by a virtualized embedded Linux Operating System (OS).

\section{ROS Environment/Interfaces}

On top of the embedded Linux we run the ROS environment to provide the processing data (audio source data and localization) to higher-level applications that perform the identification and classification of the audio events inside the vehicle.

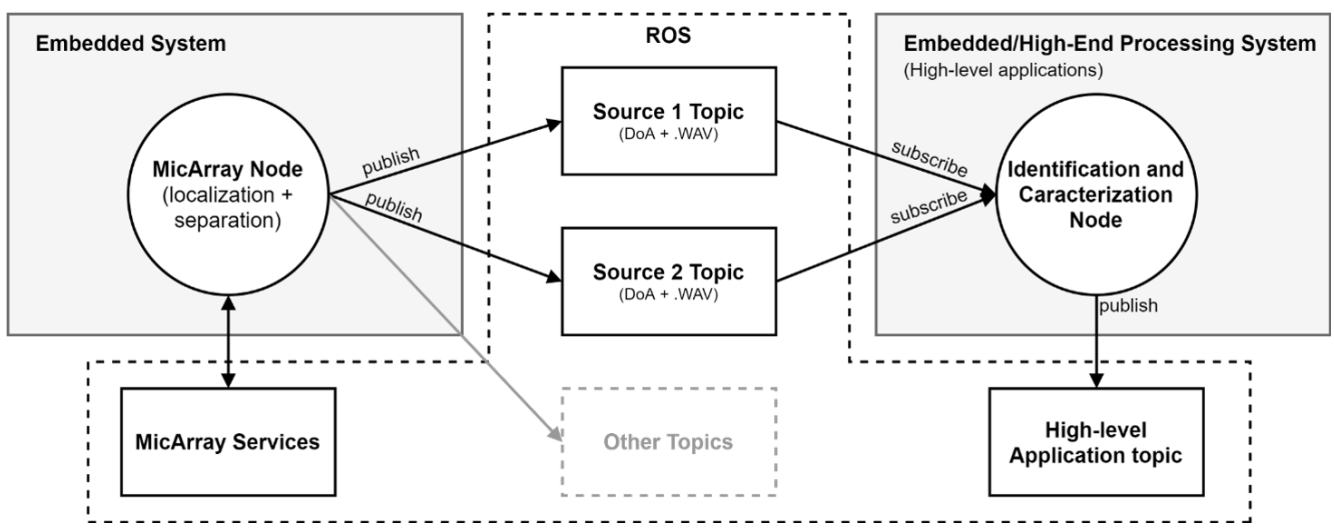

Figure 2. ROS architecture/interface between the sound source localization and separation system and the characterization algorithm.

Figure 2 depicts the ROS architecture of the microphone array for sound identification and localization. Upon the arrival of new audio data, the MicArray Node reads and processes the output from the localization algorithm in the PL, i.e., the DoA 
and the source audio sample in the WAV format, and publishes it to multiple topics according to the audio source (one topic for each source). Finally, each audio source topic is subscribed by the Identification and Characterization Node, which applies the classification and identification algorithms and forwards data to higher-level applications for further processing. Moreover, the system provides a collection of services, which act as an interface to execute several actions, e.g., perform hardware initialization, change parameters, configure the microphone array, etc.

\section{Microphone Array System}

To develop the sensor system, and to comply with the project's requirements, the microphone array must provide specific features such as: (1) have an UCA geometry with a maximum diameter of $10 \mathrm{~cm}$; (2) use up to seven low-power MEMS microphones; and (3) include an FMC interface. In contrast to linear arrays, an UCA geometry allows to use sound source localization in a 3D space, and the FMC interface allows the Printed Circuit Board (PCB) to be plugged to other platforms that support this connector. All microphones are five centimeters spaced between them, providing an UCA with six microphones placed around the circumference $\left(60^{\circ}\right.$ between microphones). Additionally, one microphone is placed in the center to be used as reference during the computation of the algorithms. The PCB layout also has six LEDs, placed around the array and parallel to the microphones, to indicate the calculated localization of the detected sound source. The FMC connector, placed at the bottom side, allows to make the direct connection of the processing platform with the microphones and the LEDs.

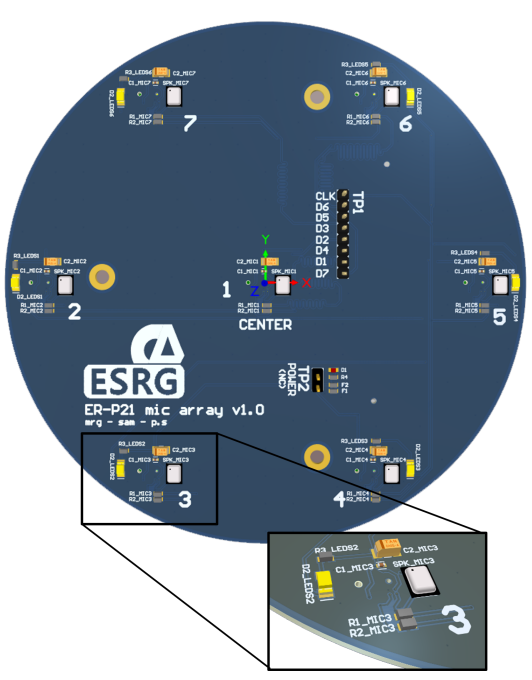

(a) Top-view.

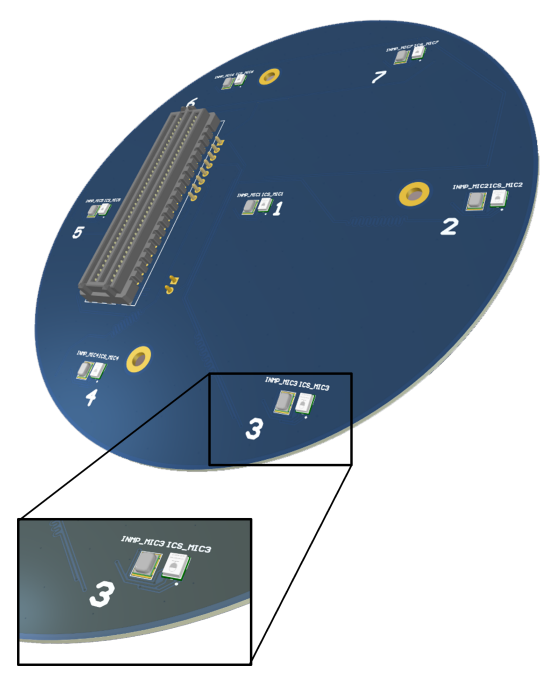

(b) Bottom-view.

Figure 3. 3D view of the microphone array PCB.

Figure 3 shows the PCB layout developed for the microphone array. For testing different microphone devices, this board supports three kind of omnidirectional and lowpower MEMS microphones: INMP621 and ICS-51360 (from TDK InvenSense) [21,22], and SPK0641HT4H-1 (from Knowles) [23]. Among other features, they all provide low-power modes, data output in PDM format, and they all work with a clock signal around 2.4 MHz. These features allows the utilization of the same controller for any board configuration. However, and to simplify the sound source localization process, on each PCB prototype it can only be used one type of microphone in the array. Due to this geometry, the maximum frequency that the system can handle is $3430 \mathrm{~Hz}$, which is the spatial aliasing frequency, $f_{\text {Spatial Aliasing, }}$ as shown in Equation 1, defined by [20]:

$$
f_{\text {Spatial Aliasing }}=\frac{c}{2 d}
$$


where $c$ is the sound speed (in this case it was considered the sound speed in the air at $20^{\circ} \mathrm{C}, 343 \mathrm{~m} \cdot \mathrm{s}^{-1}$ ), and $d$ is the smaller distance between microphones, $0.05 \mathrm{~m}$.

\section{Processing Platform}

The processing platform includes a PL system with FPGA technology, and a PS with an Application Processing Unit (APU), which are both the main units used in this prototype. The communication between both systems is achieved via the AXI protocol through the AMBA bus and by resorting to the available Direct Memory Access (DMA) controller, and the communication with the microphone array PCB is done through the FMC interface. The processing platform deploys on the PL the Signal Acquisition module (responsible of acquiring and converting the signal of each microphone) and the Signal Processing module (which includes the algorithm to localize the sound source). By its turn, the PS gives supports to the hypervisor, Linux OS, device drivers, and the ROS interfaces.

\subsection{Signal Acquisition Module}

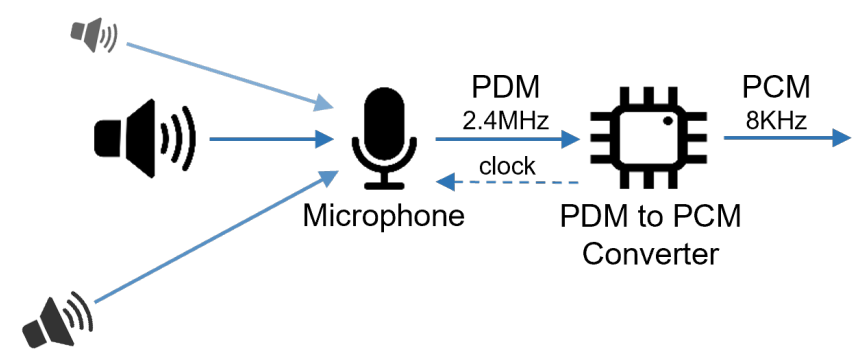

Figure 4. Data acquisition system with the PDM to PCM converter.

The data acquisition module is responsible to generate a clock signal of $2.4 \mathrm{MHz}$ to interface the microphones and to collect and convert each microphone data output from the PDM to the PCM format. Figure 4 displays the data flow between each microphone and its corresponding acquisition block. Since the microphone output is a PDM signal with a switching frequency of $2.4 \mathrm{MHz}$, the PDM to PCM converter block deployed in the FPGA is responsible for: (1) generating a clock signal at $2.4 \mathrm{MHz}$; (2) acquiring the data from the microphone at the same clock frequency; and (3) converting the signal from PDM at $2.4 \mathrm{MHz}$ to the PCM format at $8 \mathrm{kHz}$ with a 24-bit resolution.

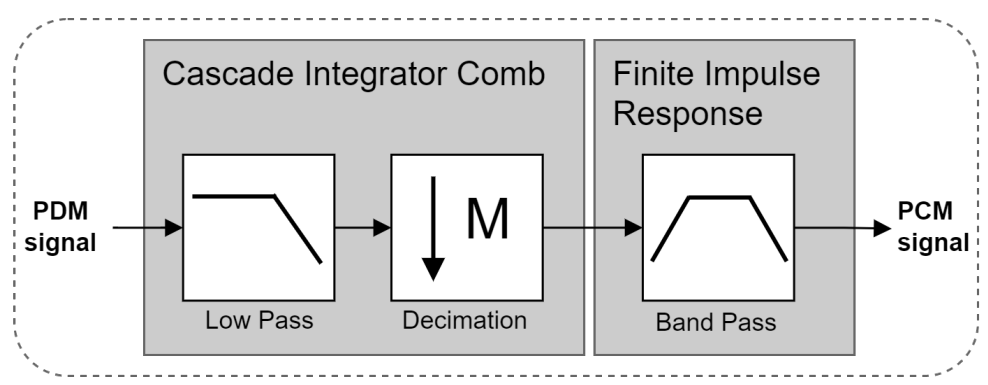

Figure 5. Block diagram of the conversion of PDM to PCM signal.

To convert the signal from PDM to PCM for each microphone [24], it was used the processing steps depicted in Figure 5. The process has three stages, starting with a lowpass filter that receives the PDM signal from the microphone and, after a quantization process, outputs a new signal to the next block. Since the microphones use a sampling frequency of $2.4 \mathrm{MHz}$, the next block performs a decimation by a factor of 300 , which creates a new signal frequency of $8 \mathrm{kHz}$. These two stages are developed in the FPGA using a Cascade Integrator Comb (CIC) filter block. The last stage corresponds to a Finite Impulse Response (FIR) filter block, that performs a band-pass filter. This filter has the 
lower cut-off frequency at $70 \mathrm{~Hz}$ to remove the Direct Current (DC) component, and it has the upper cut-off frequency at $3 \mathrm{kHz}$, which reduces possible aliasing phenomena (audio and spatial aliasing). Although the bandwidth of the pass-band filter is between $70 \mathrm{~Hz}$ and $3 \mathrm{kHz}$, this range is enough for the human voice fundamental frequency, which is commonly between 85 to $155 \mathrm{~Hz}$ for an adult male, and 165 to $255 \mathrm{~Hz}$ to an adult female [25].

\subsection{Sound Source Localization}

The algorithm to localize the sound sources is presented in Figure 6. Since its working principle is based on the signals energy, it requires data from all microphones to calculate the DoA of the sound source. This task is performed in six sequential steps: (1) Absolute Value; (2) Average Value; (3) Noise Removal; (4) Polar to Cartesian; (5) DoA Calculation; and (6) Get Angle.

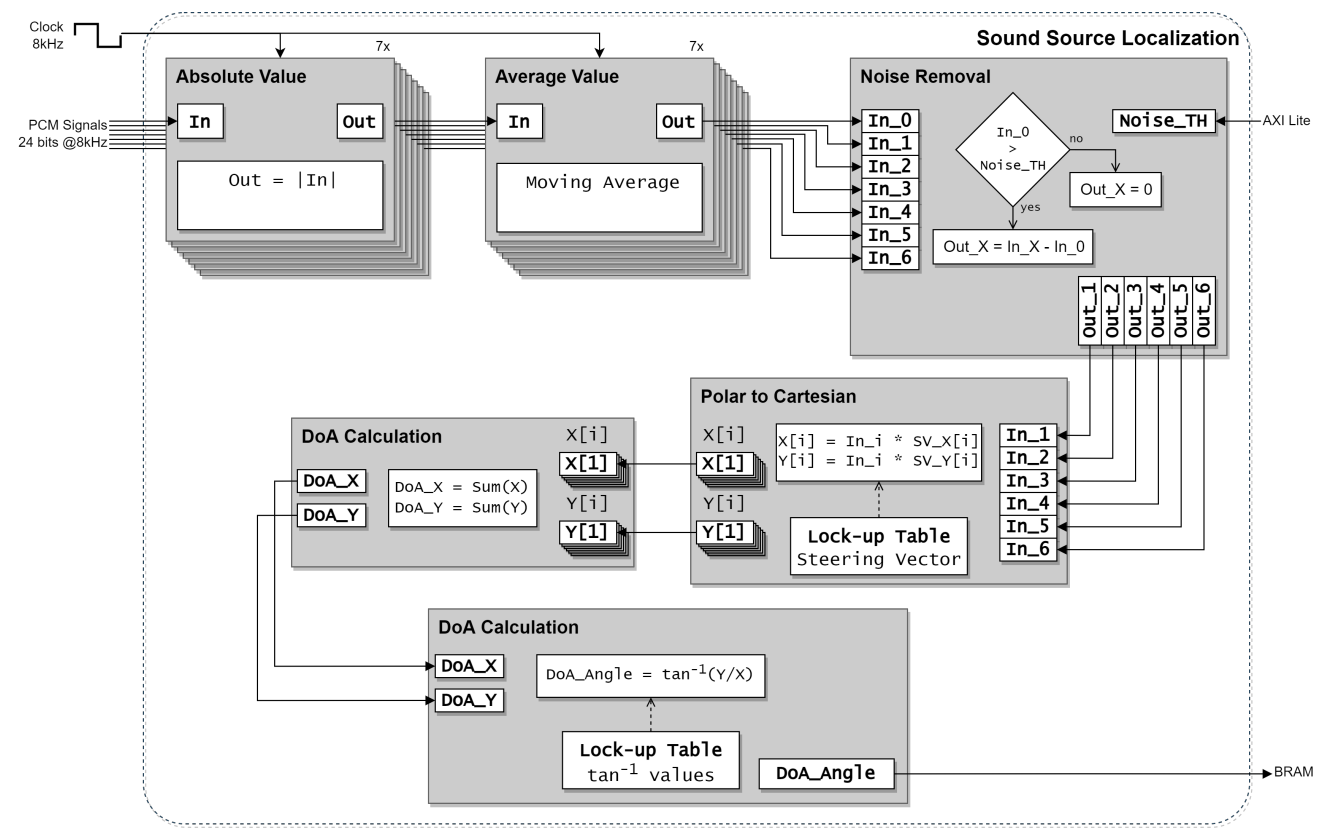

Figure 6. Block diagram of the energy sound source localization algorithm.

(1) Absolute Value: In this step, for each microphone, the input data (in PCM format) is received at the same frequency of the sampling frequency $(8 \mathrm{kHz})$ to calculate its absolute value.

(2) Average Value: This step receives data from the previous block and calculates the moving average for each microphone signal.

(3) Noise Removal: This block receives the data from the previous step and a userdefined noise threshold signal (Noise Threshold). If the average value of the central microphone is less than the Noise Threshold, then it is considered only background noise in the environment, and the new average value for each microphone is set to zero. Otherwise, the average value of the central microphone is subtracted from the remaining microphone's data.

(4) Polar to Cartesian: This stage calculates, for each of the six UCA microphones, its cartesian position multiplied by its corresponding average value. The output from this block is the weight vector for each microphone according to the signal energy.

(5) DoA Calculation: In this step, it is calculated the resultant of all vectors to output a cartesian vector with the DoA estimation.

(6) Get Angle: This stage calculates the DoA angle from the cartesian vector. 
To optimize the conversion steps, the Polar to Cartesian and the Get Angle, make use of look-up tables. Additionally, the output from the Polar to Cartesian stage provides information to control the LEDs in the microphone array. The output data corresponds to the weight of each microphone according to the location of the sound source. This data is processed to generate a Pulse-width Modulation (PWM) signal for each LED, which results in a brighter light on the LEDs closer to the sound source.

\subsection{Interface between the PL and the PS}

The communication between the PS and the PL is made through the AXI-Lite protocol over the AMBA bus. System data can be classified in: (1) data acquired by the microphones and processed in hardware that includes the DoA estimation and the respective PCM signal, transferred from the PL to the PS; and (2) control data transferred from the PS to the PL that is used to configure the acquisition and localization systems. In the first case, i.e., data from the PL to the PS, the process is performed in two ways:

(1) the PCM signal data is written to the Block Random Access Memory (BRAM) directly through a BRAM controller that defines the writing position. This data is accessed by the PS through an AXI interface connected to the BRAM;

(2) the DoA data is directly set available to the PS via AXI-Lite interface.

In the second case, where the PL receives the control signals from PS, it is used the AXI-Lite protocol, where the PS can access control registers to enable the PDM-to-PCM Converter and Signal Processing modules, and to configure the noise threshold inside the sound source localization module.

\subsection{Software Stack}

Figure 7 depicts the software stack that is supported by the PS. The ROS2 system, supported by a virtualized Linux, is used to ease the integration of the microphone array with the ROS network standard. As previously shown in Figure 2 from Section 2, it provides specific interfaces of nodes and topics, allowing both for system flexibility, scalability, and interoperability features. Moreover, to configure and capture the audio data packages from the microphone array within the OS, a device driver was developed and included in the custom Linux image. Finally, to guarantee data security and prevent unwanted accesses from third parties to the audio data that flows through the system, an additional virtualization layer was added through our in-house made static partitioning hypervisor Bao [26].

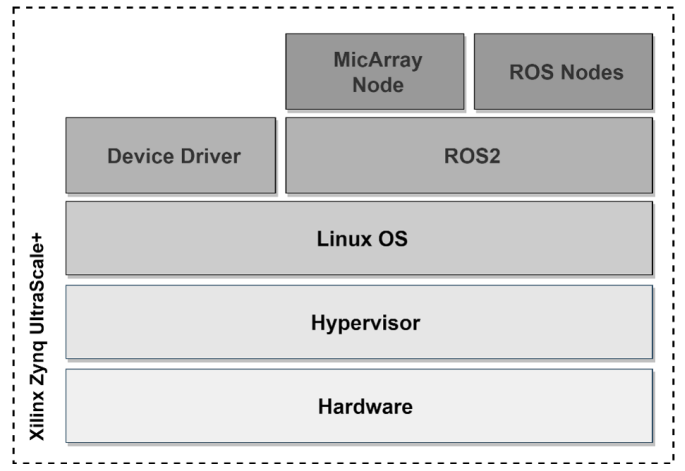

Figure 7. System software stack.

\section{Results}

In order to test and evaluate the speaker localization and identification prototype, we have used the following experimental setup: (1) the sensor system prototype (Figure 8a); (2) an audio sound source; and (3) a laptop computer with a ROS interface that subscribes to the ROS topics. The laptop is connected to the processing platform through an Ethernet interface (in a wired ad-hoc network using an SSH session) to control the 
prototype system and store the acquired and processed data (signal data and DoA from each sound source). Regarding the sensor system, two different steps were executed to demonstrate its behaviour with just one sound source:

(1) first step test evaluates and verifies the acquisition system, i.e., sampling, PDM to PCM format conversion, and data filtering;

(2) second step evaluates the localization system in the presence of a moving sound source, checking the DoA and verifying the resulting angle with the actual position of the sound source.

The center microphone is used as the reference to calculate the DoA, and the angle measurements start at $0^{\circ}$ on the positive $x$-axis of the unit circle graph (corresponding to the microphone number 5), and go counter-clockwise around the circle until they are back at $360^{\circ}$ (Figure $8 \mathrm{~b}$ ). Since the sensor system is to be placed in the center of the vehicle's roof and to bring the sound sources closer to the conventional passenger locations, during the tests, the sound source was placed between 50 to $100 \mathrm{~cm}$ away from the microphone array with an elevation of $45^{\circ}$.

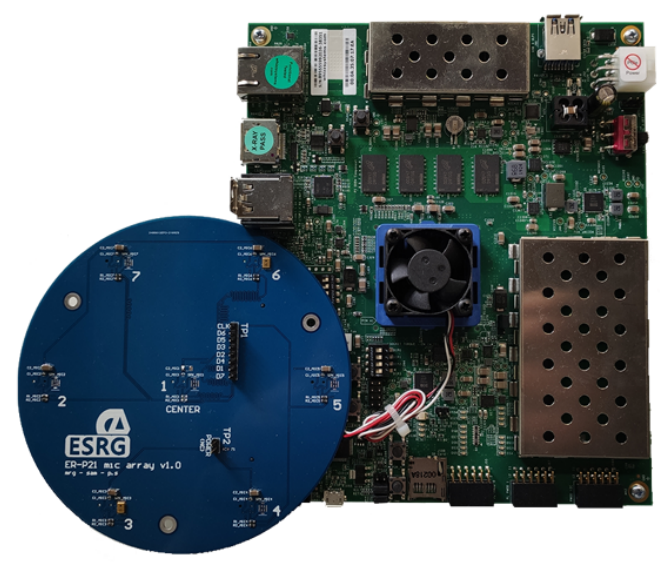

(a) Sensor system prototype.

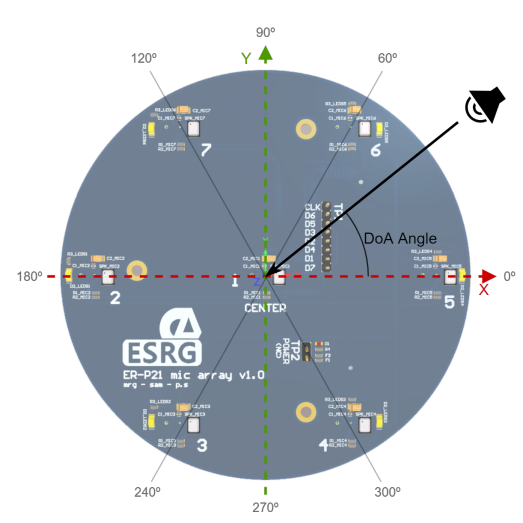

(b) Microphone's location on a XY referential.

Figure 8. Sensor system prototype to localize and identify sound sources in an SAV and location of each microphone in the PCB to calculate the DoA.

\subsection{Data Acquisition}

To test the acquisition modules, the system was adapted to bypass the sound source localization module and each microphone's data is directly sent from the PDM-to-PCM module to the device driver using the BRAM controller. During the tests, the sensor system was exposed to different sound sources, which allowed to analyse the behaviour of the PDM-to-PCM module at different frequencies and distances. Figure 9 depicts the results for the three types of microphones available in the microphone array. The sound source used to test the data acquisition was a controlled signal (sine wave) at different frequencies: $220 \mathrm{~Hz}$ (Figure 9a); $440 \mathrm{~Hz}$ (Figure 9b), which is currently used as the reference frequency for tuning musical instruments; $880 \mathrm{~Hz}$ (Figure 9c); and 1760 $\mathrm{Hz}$ (Figure 9d). All the sound sources have their fundamental frequency within the bandwidth defined for the band-pass filter. 


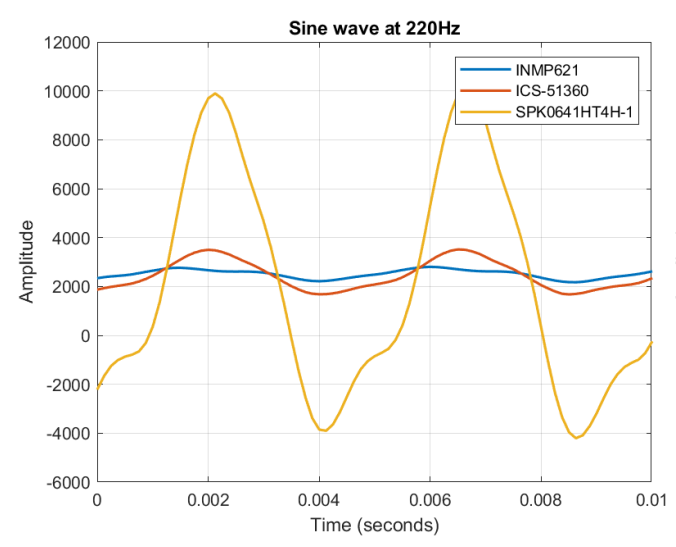

(a) Sine wave at $220 \mathrm{~Hz}$.

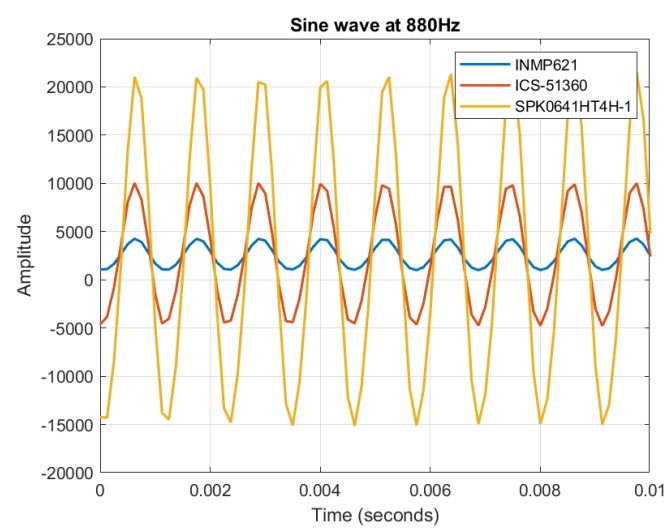

(c) Sine wave at $880 \mathrm{~Hz}$.

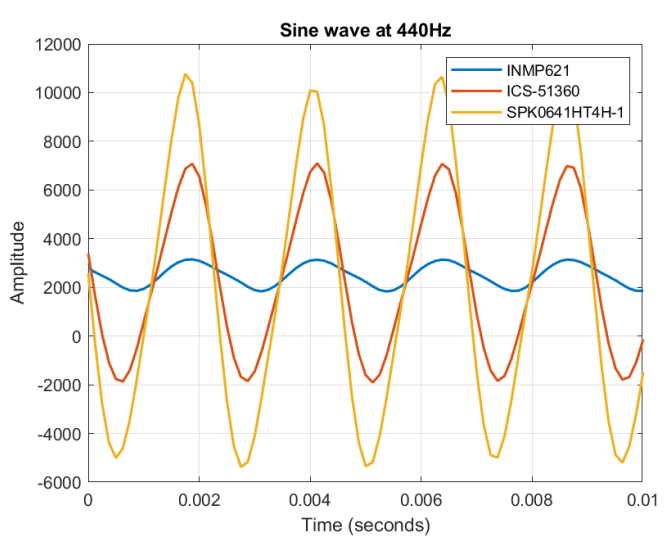

(b) Sine wave at $440 \mathrm{~Hz}$.

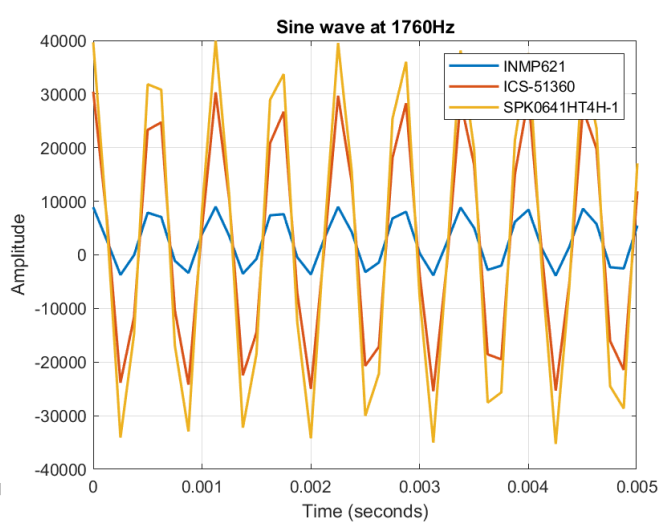

(d) Sine wave at $1760 \mathrm{~Hz}$.

Figure 9. Data acquisition by the three types of microphones at different frequencies.

The results show that the microphones and the conversion module can achieve a good performance in collecting sound within the frequency ranges defined by the band-pass filter since the main characteristics of the original signals are present on the acquired samples. However there are some differences in the received signal's amplitude, which are mainly associated with the receiving power of the collected signal, which can be affected by: (1) the location and position of the microphones in the array; (2) the aperture size of the microphone's sound port; (3) and the aperture size of the PCB hole for the sound port. For instance, the INMP621 and the ICS-51360 are located in the bottom layer of the microphone array, which result in signals with lower amplitude values. Moreover, Figure 9a presents some signal's distortion and lower amplitudes, which are mainly due to the speaker's ability to generate lower frequencies.

For testing the band-pass filter we have generated sound waves at frequencies above the filter's cutoff frequency which is $3 \mathrm{KHz}$. Figure 10 shows the results in each microphone type for two different frequencies, $3520 \mathrm{~Hz}$ and $7040 \mathrm{~Hz}$. When the frequency is nearby the cutoff frequency $(3 \mathrm{KHz})$, the sound is attenuated according to the low-pass component of the filter. However, at $3520 \mathrm{~Hz}$, there are still some signal components since, by definition, at the cutoff frequency the output drops below $70.7 \%$ of its input. For the $7040 \mathrm{~Hz}$ frequency, there is only noise and the audio signal is nearly zero. These results show the correct operation of the band-pass filter which is used to avoid temporal and spatial aliasing. 


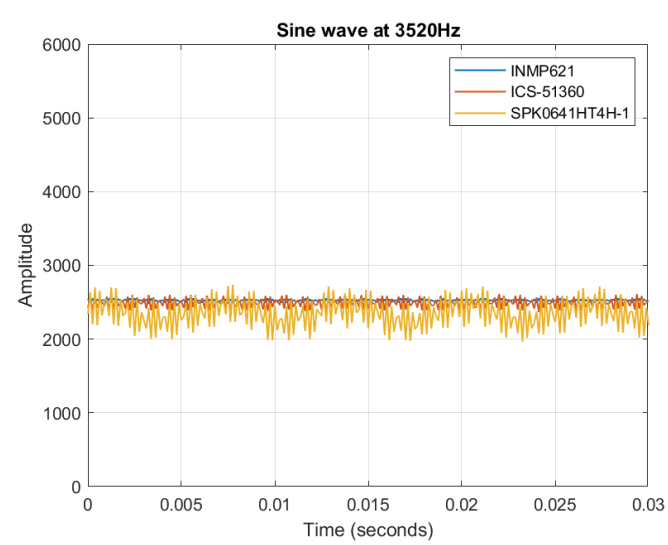

(a) Sine wave at $3520 \mathrm{~Hz}$.

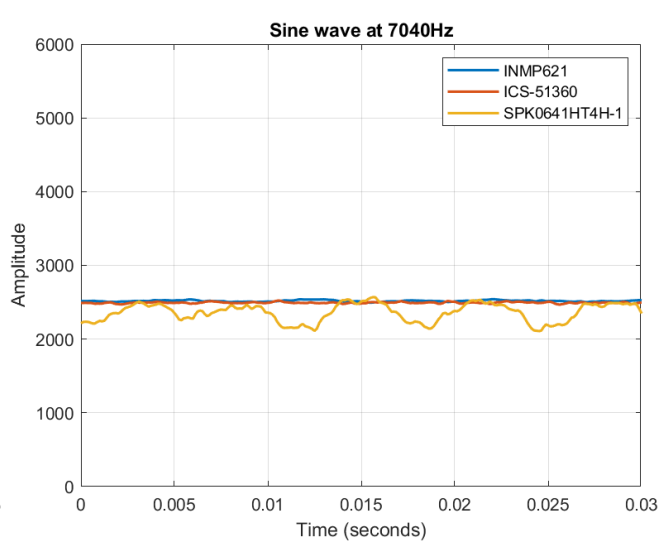

(b) Sine wave at $7040 \mathrm{~Hz}$.

Figure 10. Sine wave acquisition for frequencies above the filter's passband.

\subsection{Sound Source Localization}

To evaluate the localization process, which is the most important goal of this project, the system was tested with a moving sound source around the microphone array that followed the pattern shown in Figure 11a. The result presented in Figure $11 \mathrm{~b}$ demonstrates with precision the location of the moving sound source. Because the algorithm needs 64 samples to localize the sound source and the sampling frequency is $8 \mathrm{kHz}$, the tracking of the sound source takes around $8 \mathrm{~ms}$ to process.
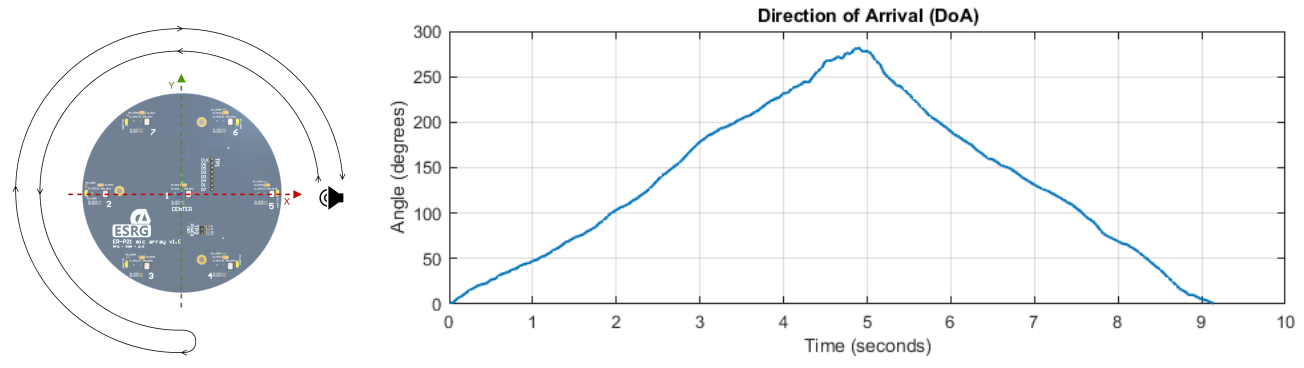

(a) Sound source path.

(b) Direction of arrival of a sound source over the time.

Figure 11. Moving sound pattern and respective calculated DoA.

\subsection{FPGA Hardware Resources}

Table 1: FPGA resources utilization.

\begin{tabular}{cccc}
\hline Resource & Utilization & Available & Utilization (\%) \\
\hline LUT & 18628 & 230400 & $8.09 \%$ \\
LUTRAM & 4550 & 101760 & $4.47 \%$ \\
FF & 21725 & 460800 & $4.71 \%$ \\
BRAM & 191 & 312 & $61.22 \%$ \\
DSP & 49 & 1728 & $2.84 \%$ \\
IO & 14 & 360 & $3.89 \%$ \\
BUFG & 5 & 544 & $0.92 \%$ \\
MMCM & 1 & 8 & $12.50 \%$ \\
\hline
\end{tabular}

The FPGA implementation requires the resources described in Table 1. In terms of LookUp Table (LUT) and LUTRAM, the implementation uses $8.09 \%$ and $4.47 \%$ of the resources available in the platform, which corresponds to 18628 LUT and 4450 LUTRAM. From the available 21725 Flip Flop (FF) units, the system uses a total of 21725 FF, which corresponds to $4.71 \%$ of the available resources. The BRAM module is the most used resource, requiring a total of 191 BRAM units corresponding to $61.22 \%$ of the available BRAM in the platform. Due to the acquisition system that using the FIR and CIC blocks, 
the system requires 49 of the available 1728 Digital Signal Processor (DSP) units (2.84\%). The hardware also requires 14 Input/Output (IO) pins, which corresponds to the 7 microphone inputs, 1 output for the clock signal used to drive the microphones, and 6 outputs to control the LEDs PWM signal. Finally, to support the clock generator module, the system requires 1 of the 8 available Mixed-Mode Clock Manager (MMCM) units, and 5 of the 544 existing Global Clock Buffer (BUFG) blocks.

\section{Conclusion}

This article presents a sensor system solution to monitor sound events in an SAV cabin. This solution is composed by a microphone array connected to a processing platform, which provides the localization of the sound sources to higher-level application through a ROS interface.

Regarding the proposed solution and the tests performed, the implemented system is able to acquire data from all microphones, filter the collected signals, and calculate the DoA of one sound source. Through individual ROS topics the MicArray Node makes the acquired audio samples available to other high-level applications, in order to identify and classify the sound events. This way, it is possible to identify the type of event that occurs and act accordingly. With respect to the architecture, the system allows the deployment of independent hardware blocks for customization and acceleration purposes. In a broader perspective, this solution, as a concept, can be integrated in other applications beyond SAV, where the localization of a sound source in a real-time approach is a major priority.

Current work encompasses the exploration of more advanced and efficient algorithms to localize and separate multiple sound source, such as variable step-size least mean square for the localization process and independent component analyses with fast convergence to separate sound sources. Furthermore, the sensor system also needs to be tested in a situation closed to the SAV reality, e.g., inside a vehicle's roof.

Acknowledgments: This work is supported by: European Structural and Investment Funds in the FEDER component, through the Operational Competitiveness and Internationalization Programme (COMPETE 2020) [Project $\mathrm{n}^{\circ}$ 039334; Funding Reference: POCI-01-0247-FEDER039334].

Conflicts of Interest: The authors declare no conflict of interest.

\section{References}

1. Litman, T. Autonomous vehicle implementation predictions; Victoria Transport Policy Institute Victoria, Canada, 2021.

2. Daily, M.; Medasani, S.; Behringer, R.; Trivedi, M. Self-Driving Cars. Computer 2017, 50, 18-23.

3. Badue, C.; Guidolini, R.; Carneiro, R.V.; Azevedo, P.; Cardoso, V.B.; Forechi, A.; Jesus, L.; Berriel, R.; Paixão, T.M.; Mutz, F.; de Paula Veronese, L.; Oliveira-Santos, T.; De Souza, A.F. Self-driving cars: A survey. Expert Systems with Applications 2021, 165, 113816.

4. Rojas-Rueda, D.; Nieuwenhuijsen, M.J.; Khreis, H.; Frumkin, H. Autonomous vehicles and public health. Annual review of public health 2020, 41, 329-345.

5. Chaudhry, B.; El-Amine, S.; Shakshuki, E.; others. Passenger safety in ride-sharing services. Procedia computer science 2018, 130, 1044-1050.

6. Fouad, R.M.; Onsy, A.; Omer, O.A. Improvement of Driverless Cars' Passengers on Board Health and Safety, using Low-Cost Real-Time Heart Rate Monitoring System. 2018 24th International Conference on Automation and Computing (ICAC), 2018, pp. $1-6$.

7. Koojo, I.; Machuve, D.; Mirau, S.; Miyingo, S.P. Design of a Passenger Security and Safety System for the Kayoola EVs Bus. 2021 IEEE AFRICON, 2021, pp. 1-6.

8. Costa, M.; Oliveira, D.; Pinto, S.; Tavares, A. Detecting Driver's Fatigue, Distraction and Activity Using a Non-Intrusive Ai-Based Monitoring System. Journal of Artificial Intelligence and Soft Computing Research 2019, 9, 247-266.

9. Pieropan, A.; Salvi, G.; Pauwels, K.; Kjellström, H. Audio-visual classification and detection of human manipulation actions. 2014 IEEE/RSJ International Conference on Intelligent Robots and Systems. IEEE, 2014, pp. 3045-3052.

10. Hulsebos, E.; Schuurmans, T.; de Vries, D.; Boone, R. Circular Microphone Array for Discrete Multichannel Audio Recording. Audio Engineering Society Convention 114. Audio Engineering Society, 2003. 
11. Tamai, Y.; Kagami, S.; Amemiya, Y.; Sasaki, Y.; Mizoguchi, H.; Takano, T. Circular microphone array for robot's audition. SENSORS, 2004 IEEE. IEEE, 2004, pp. 565-570.

12. Busso, C.; Hernanz, S.; Chu, C.W.; Kwon, S.i.; Lee, S.; Georgiou, P.G.; Cohen, I.; Narayanan, S. Smart room: Participant and speaker localization and identification. Proceedings.(ICASSP'05). IEEE International Conference on Acoustics, Speech, and Signal Processing, 2005. IEEE, 2005, Vol. 2, pp. ii-1117.

13. Chen, X.; Shi, Y.; Jiang, W. Speaker tracking and identifying based on indoor localization system and microphone array. 21st International Conference on Advanced Information Networking and Applications Workshops (AINAW'07). IEEE, 2007, Vol. 2, pp. 347-352.

14. Butt, U.M.; Khan, S.A.; Ullah, A.; Khaliq, A.; Reviriego, P.; Zahir, A. Towards Low Latency and Resource-Efficient FPGA Implementations of the MUSIC Algorithm for Direction of Arrival Estimation. IEEE Transactions on Circuits and Systems I: Regular Papers 2021.

15. Da Silva, B.; Braeken, A.; Touhafi, A. FPGA-based architectures for acoustic beamforming with microphone arrays: trends, challenges and research opportunities. Computers 2018, 7, 41.

16. Jung, Y.; Jeon, H.; Lee, S.; Jung, Y. Scalable ESPRIT Processor for Direction-of-Arrival Estimation of Frequency Modulated Continuous Wave Radar. Electronics 2021, 10, 6952021.

17. Nsalo Kong, D.F.; Shen, C.; Tian, C.; Zhang, K. A New Low-Cost Acoustic Beamforming Architecture for Real-Time Marine Sensing: Evaluation and Design. Journal of Marine Science and Engineering 2021, 9, 868.

18. Ribeiro, Â.; Rodrigues, C.; Marques, I.; Monteiro, J.; Cabral, J.; Gomes, T. Deploying a Real-Time Operating System on a Reconfigurable Internet of Things End-device. IECON 2019-45th Annual Conference of the IEEE Industrial Electronics Society. IEEE, 2019, Vol. 1, pp. 2946-2951.

19. Marques, I.; Rodrigues, C.; Tavares, A.; Pinto, S.; Gomes, T. Lock-V: A heterogeneous fault tolerance architecture based on Arm and RISC-V. Microelectronics Reliability 2021, 120, 114120.

20. Brandstein, M.; Ward, D.; Lacroix, A.; Venetsanopoulos, A., Eds. Microphone Arrays: Signal Processing Techniques and Applications, 1st ed.; Digital Signal Processing, Springer Berlin Heidelberg: Berlin, Heidelberg, 2001.

21. InvenSense. Wide Dynamic Range Microphone with PDM Digital Output Data Sheet ADMP62. DS-INMP621-00 Datasheet Rev 1.32016.

22. InvenSense. Bottom Port PDM Digital Output Multi-Mode Microphone. ICS-51360 Datasheet Rev 1.0 2016.

23. Knowles. Digital SiSonic Microphone With Multiple Performance Modes. Datasheet SPK0641HT4H-1 Rev A 2016.

24. Hegde, N. Seamlessly interfacing MEMs microphones with blackfin processors. EE-350 Engineer-to-Engineer Note 2010.

25. Re, D.E.; O'Connor, J.J.; Bennett, P.J.; Feinberg, D.R. Preferences for very low and very high voice pitch in humans. PloS one 2012, 7, e32719.

26. Martins, J.; Tavares, A.; Solieri, M.; Bertogna, M.; Pinto, S. Bao: A lightweight static partitioning hypervisor for modern multi-core embedded systems. Workshop on Next Generation Real-Time Embedded Systems (NG-RES 2020). Schloss Dagstuhl-LeibnizZentrum für Informatik, 2020. 